\title{
RECHTSSPRACHE UND RECHTSINFORMATIK IM EUROPÄISCHEN MEHREBENENSYSTEM
}

\author{
Günther Schefbeck
}

Abstract: Das Problem des sprachlichen Zugangs zum Recht stellt sich im europäischen Mehrebenensystem, bedingt durch das Erfordernis die nationalen Jurisdiktionen übergreifender rechtssprachlicher Abstraktion und die Herausforderung der Multilingualität, neu. In der Überlagerung europäischer und nationaler Rechtsetzung wirkt die Resultante der rechtssprachlichen Entwicklung mehr divergent als konvergent. Keine Rücksicht nimmt die europäische Rechtssprache auf die Besonderheit plurizentrischer Sprachen, zu zeigen am österreichischen Deutsch. Daraus ergeben sich neue Aufgabenstellungen für die Rechtsinformatik.

\section{Inhaltsverzeichnis}
A. Einleitung. 37
B. Die Sprache des Gesetzgebers 40
C. Nationale rechtssprachliche Divergenz 43
D. Die Sprache(n) des europäischen Rechts 48
E. Rechtssprache(n) zwischen Konvergenz und Divergenz 51
F. Aufgaben der Rechtsinformatik . .58

\section{A. EINLEITUNG}

Der Rechtsgelehrte in seiner gesellschaftlichen Verantwortung: Unter den Stellungnahmen zum Ministerialentwurf der Urheberrechtsgesetz-Novelle 2015 findet sich auch eine von Erich Schweighofer - bezeichnenderweise von Los Angeles aus - abgegebene und darin wiederum der die legistische Gestaltung dieses Rechtsaktsentwurfs vernich- 
tend beurteilende Satz: «Das Recht der Bürgerinnen und Bürger auf eine klare und verständliche Rechtssprache wird verletzt.»1

Nun ist, wie wir wissen, ein solches Recht zwar mit gutem Grund zu postulieren, aber schwer zu positivieren. Die viel zitierte Forderung des Codex Iustinianus, wonach «leges ab omnibus intellegi debent» ${ }^{2}$, erscheint als Vorbedingung für die in den real existierenden Rechtsordnungen ${ }^{3}$ regelmäßig verankerte Vermutung der Rechtskenntnis bzw. der Kenntnis der gehörig kundgemachten Rechtsvorschriften - allein die Kontrolle, ob diese Vorbedingung erfüllt sei, ist in der Regel eine nachgängige und überdies nur punktuell wirksam werdende. Schon lange vor seinem «Denksporterkenntnis» ${ }^{4}$ hat der österreichische Verfassungsgerichtshof, ausgehend vom Publizitätsgebot der Art. 49 und 97 B-VG, grundsätzlich erkannt: «Wenn der Zweck der Rechtsordnung darin besteht, den Menschen durch die Vorstellung der Norm zu einem normgemäßen Verhalten zu veranlassen, so ist der Gesetzgeber gehalten, die Möglichkeit einer solchen Vorstellung zu geben ... Eine Vorschrift, zu deren Sinnermittlung subtile verfassungsrechtliche Kenntnisse, qualifizierte juristische Befähigung und Erfahrung und geradezu archivarischer Fleiß von Nöten sind, ist keine verbindliche Norm.»5

Wie Fred Rodell, «enfant terrible» der US-amerikanischen Rechtswissenschaft, schon vor einem hohen Menschenalter generell festgestellt hat: «There are two things wrong with almost all legal writing. One is its style. The other is its content.»6 Mag der Inhalt der Rechtsvorschriften durch konfligierende Interessen und notwendige Kompromissbildung bestimmt und damit zwar der rechtspolitischen, nicht aber der legistischen

1 Stellungnahme 94/SN-132/ME-XXV. GP vom 14.06.2015, https://www.parlament.gv.at/PAKT/ VHG/XXV/SNME/SNME_04184/imfname_423589.pdf.

2 Codex Iustinianus 1, 14, 9.

3 So in der österreichischen insb. in §§ 2 ABGB bzw. 9 StGB.

4 VfSlg. 12420/1990. Vgl. dazu z.B. Clemens JABLoneR, Das «Denksporterkenntnis» des Verfassungsgerichtshofes im Spannungsfeld von Verfassungsrecht und Rechtstechnik, in: BERNDChristian FunK u.a. (Hg.), Festschrift für Ludwig Adamovich zum 60. Geburtstag, Wien 1992, S. 189-201.

5 VfSlg. 3130/1956.

6 Fred Rodell, Goodbye to Law Reviews, in: Virginia Law Review 23 (1936/37), S. 38-45, hier S. 38 . 
Kritik auszusetzen sein, so muss der «Stil», in unserem Zusammenhang also die formale systematische und sprachliche Gestaltung der Rechtsvorschriften, den für die Legistik festgelegten Standards entsprechen und daher einer systematischen Legistikkritik unterzogen werden. Das ist Aufgabe engagierter Rechtsgelehrter wie des Jubilars, aber natürlich auch der organisierten und nicht organisierten Zivilgesellschaft. Darin auch eine primäre Aufgabe des Parlaments zu sehen, ist angesichts der in der modernen parteienstaatlichen Demokratie überwiegend bei der Regierung liegenden Gesetzesinitiative oft erhobene Forderung, welcher in manchen parlamentarischen Systemen unter anderem dadurch Rechnung getragen wird, dass sich das Parlament rechtslinguistischer Expertise bedient ${ }^{7}$. Dass dem österreichischen Parlament solche Expertise vorenthalten bleibt, mag mit ursächlich für den Eindruck sein, die österreichische Rechtsetzung würde jenen Grundsätzen, welche die Legistischen Richtlinien gleich eingangs für die sprachliche Gestaltung von Rechtsvorschriften, insbesondere hinsichtlich ihrer «Verständlichkeit»», festlegen, nicht gerecht. Vom Grundsatz zu seiner Operationalisierung als Voraussetzung für seine Realisierung ist der Weg oft weit.

Seit Fritz Schönherr in seinen bereits klassisch zu nennenden Arbeiten die sprachliche Qualität der österreichischen Rechtsvorschriften systematisch analysiert und kritisiert hat $^{9}$, seit Friedrich Lachmayer in Reaktion darauf den absurd-realistischen Verfahrensvorschlag geäußert hat, die geschäftsordnungsrechtlich vorgesehenen drei Lesungen von Gesetzesvorschlägen im Nationalrat wörtlich zu nehmen und so einen Anreiz für rechtssprachliche Disziplinierung zu schaffen ${ }^{10}$, seit also im Blick auf die österreichische Rechtsetzung Fragen der Qualität der Rechtssprache und ihrer Verbesserung in

7 So steht gemäß § 80a GO-BT dem Deutschen Bundestag seit 1966 ein Redaktionsstab zur Seite, der im Auftrag der Ausschüsse Gesetzentwürfe «auf sprachliche Richtigkeit und Verständlichkeit» zu prüfen hat. Die politisch besetzte, aber gemäß Art. 7 lit. b GOPD mit Fachexpertise ausgestattete Redaktionskommission der eidgenössischen Räte hat die weiter gehende Funktion, die endgültige Sprachfassung der «Erlasse» für die Schlussabstimmung festzulegen.

$8 \quad$ Z 9 LegRL 1990, https://www.justiz.gv.at/file/2c94848a60c158380160e4e3747c0c33.de.0/ legrl1990.pdf.

9 Siehe insb. Fritz SchÖnhERR, Sprache und Recht. Aufsätze und Vorträge, Wien 1985, weiters Theo ÖHLInger (Hg.), Recht und Sprache. Fritz Schönherr-Gedächtnissymposium 1985, Wien 1986.

10 FRIEDRICH LACHMAYER, Ein Verfahrensvorschlag für verständlichere Gesetze, in: Österreichisches Anwaltsblatt 1988, S. 325f. 
den 1970er und 1980er Jahren breiter diskutiert worden sind, haben sich die Rahmenbedingungen der Rechtsetzung grundlegend verändert. In der supranationalen Gemeinschaft der Europäischen Union, im politischen und rechtlichen «Mehrebenensystem»11, erscheint nicht nur die Systematik der Rechtsordnung durch Überschichtung komplexer als innerhalb einer nationalstaatlichen Jurisdiktion, sondern auch die Rechtssprache spiegelt den multilingualen Anspruch der Gemeinschaft wider. Und so erscheint ein altes Problem in neuem Licht.

\section{B. DIE SPRACHE DES GESETZGEBERS}

«Der Gesetzgeber soll denken wie ein Philosoph, aber reden wie ein Bauer,» lautet die klassische Forderung an die Legislative, die in Deutschland zumeist entweder Rudolf von Ihering oder Gustav Radbruch, in der Schweiz in der Regel Eugen Huber zugeschrieben wird; in Österreich werden keine eigenen urheberrechtlichen Ansprüche erhoben, die Aussage aber wird nicht minder häufig zitiert. Der Gesetzgeber also soll: denken und - reden. Das Denken des Gesetzgebers, das sich in der Dialektik des vorparlamentarischen und des parlamentarischen Diskurses manifestiert, ist ein Thema für sich; hier soll uns das Reden des Gesetzgebers interessieren.

Das Reden, auch des Gesetzgebers, vollzieht sich primär im Medium der Sprache. Mag Sprache auch bildhaft sein, sie bleibt dem - ursprünglich gesprochenen, gerade in der Gesetzgebung seit langem aber schriftlich festgehaltenen - Wort verhaftet. Mag das gesprochene Wort durch seine Intonation und durch die es begleitende Gestik erst seine Kontextualisierung erfahren und damit eine wesentliche Voraussetzung für seine Interpretation geschaffen werden, so entbehrt das geschriebene Wort dieser Interpretationshilfe. Fachsprachen wie die juristische werden daher, um die Wahrscheinlichkeit konvergenter Auslegung der in ihnen zum Ausdruck gebrachten Inhalte zu erhöhen, durch ein umfangreiches Instrumentarium von Interpretationsmethoden begleitet, Fachtexte,

11 Vgl. zum Konzept z.B. Johannes Huinink, Mehrebenensystem-Modelle in den Sozialwissenschaften, Wiesbaden 1989, zur Anwendung des Modells auf die Europäische Union THOMAS KöNIG u.a. (Hg.), Das europäische Mehrebenensystem, Frankfurt a.M. 1996, mit speziellem, zum Zeitpunkt des Erscheinens noch geradezu aufklärerischem Bezug zu Österreich GERDA FALKNER/WOLFGANG C. MÜLLER (Hg.), Österreich im europäischen Mehrebenensystem, Wien 1998. 
wie es Rechtsnormen sind, oftmals durch «erläuternde» Texte, deren Spektrum in unserer normativen Textsorte von die Motive der rechtsetzenden Autorität zum Ausdruck bringenden Präambeln bis zu nichtnormativen historischen Materialien reicht ${ }^{12}$; Texte sollen also Texte interpretieren helfen, was in einen unendlichen Regress des Interpretationsproblems mündet, der nur durch Kontextualisierung durchbrochen werden kann.

Die supranationale Rechtsetzung der Europäischen Union hat dieses Problem neu dimensioniert. Europäische Rechtsnormen werden in der Regel in einer Arbeitssprache allenfalls parallel in einer zweiten - konzipiert und verhandelt, aber in allen Amtssprachen kundgemacht und gelten als in ihnen gleichermaßen verbindlich ${ }^{13}$. Somit stellt sich ganz unmittelbar, und mit größter praktischer Relevanz, die Frage nach der Möglichkeit äquivalenter Sprachtransformationen, also die Frage: Kann gewährleistet werden, dass die Bedeutung einer textuellen Aussage bei ihrer Übersetzung von einer Sprache in eine andere (analog aber natürlich auch schon bei ihrer Übersetzung von einer Formulierung in eine andere innerhalb ein und derselben Sprache) erhalten bleibt?

Was aber ist die «Bedeutung»? Schon bei der Unbestimmtheit des Bedeutungsbegriffs setzt Quine an, wenn er die These von der Unbestimmtheit der Übersetzung entwickelt ${ }^{14}$. Kann aber der Bedeutungsbegriff immerhin noch empirisch gefasst werden Quine bezieht ihn bei Beobachtungsbegriffen auf die jeweils ausgelösten Reize, also auf die Sinneseindrücke, bei theoretischen Begriffen auf die jeweiligen empirischen Konsequenzen -, so scheitert der Versuch, der Übersetzung Bestimmtheit zu verleihen, an der oft wahrnehmbaren Mehrzahl möglicher empirischer Äquivalenzen: Begriffe und ihre Ausdrücke in einer Sprache stehen zumeist nicht in ein-eindeutigen Beziehungen zu einander, und für Begriffe in unterschiedlichen Sprachen gilt das umso mehr. Mehr

12 Vgl. z.B. Clemens JabloneR, Die Gesetzesmaterialien als Mittel der historischen Auslegung, in: HANS WALTHER KalUZA u.a. (Hg.), Für Staat und Recht. Festschrift für Herbert Schambeck, Berlin 1994, S. 441-457.

13 Siehe insb. EuGH, Rs. 283/81, Entscheidungsgrund 18.

14 Dazu grundlegend sein Hauptwerk: WILLARD VAN ORMAN QUINE, Word and Object, new ed., Cambridge, Mass., 2013, insb. S. 23-71. Einen Überblick gibt ALAN WEIR, Indeterminacy of Translation, in: ERNEST LEPORE/BARRY C. SMITH (Hg.), The Oxford Handbook of Philosophy of Language, Oxford 2006, S. 233-249; vgl. auch EvE GAUDET, Quine on Meaning, London 2006. 
noch: Begriffe und ihre Ausdrücke sind eingebettet in eine komplexe, überdies von stetem Wandel des Sprachgebrauchs beeinflusste Bedeutungslandschaft; die geradezu universelle Polysemie wird noch unterfüttert durch zumindest konnotative Divergenz in den verschiedenen, einander überlagernden Lekten, von den Regiolekten über die Soziolekte bis hin zu Idiolekten.

Die Rechtssprache kann in mehrfacher Hinsicht als ein Soziolekt aufgefasst werden: Zum einen ist sie die Sprache einer am Recht geschulten sozialen Gruppe und reproduziert sich insofern selbstreferentiell mit der Tendenz, sich von anderen Soziolekten oder dem empirisch nur schwer fassbaren Konstrukt der «Alltagssprache» zu entfernen, wenn dieser Tendenz nicht proaktiv rechtspolitisch entgegengewirkt wird; die «plain language»-Bewegung in der angelsächsischen Welt ist der wirkmächtigste derartige Versuch in der jüngeren Vergangenheit ${ }^{15}$. In der Regel redet der Gesetzgeber nicht wie ein Bauer, sondern wie ein Jurist.

Zum anderen ist die Rechtssprache gebunden an ein makrosoziales System: Seit der frühen Neuzeit ist das der Territorial-, unter dem fortwirkenden Einfluss des 19. Jahrhunderts auch heute noch primär der Nationalstaat. Selbst Staaten, in denen, aus der linguistischen Ferne betrachtet, die «gleiche Sprache» gesprochen wird - und die sich unter Umständen sogar ganz bewusst um konvergente Sprachfortbildung bemühen mögen, wie dies Deutschland, Österreich und die Schweiz vermittels ihrer Rechtschreibkommissionen getan haben -, haben jeweils ganz unterschiedliche Rechtssprachen ausgebildet, was auch gar nicht überraschen kann, spiegeln diese doch die je unterschiedlichen normativen Konzepte wider, die ihrerseits wiederum die je unter-

Siehe insb. Plain Writing Act of 2010, H.R. 946, Publ.L. 111-274, sowie die dazu ergangenen Executive Orders 12866, 12988 und 13553. Vgl. aus der Fülle der dazu verfügbaren legistischen Fachliteratur z.B. BRYAN A. GARNER, Legal Writing in Plain English: A text with exercises, 2. Aufl., Chicago 2013; PETER BuTT, Modern Legal Drafting: A guide to using clearer language, 3. Aufl., Cambridge 2013. Sind die USA unter der Obama-Administration auch der Ausgangspunkt für die Ausbreitung des «plain language»-Konzepts in der Rechtssprache gewesen, so sind solche Ansätze, mit je unterschiedlichem Grad von Verbindlichkeit, heute in vielen normativen Systemen zu finden. Für Österreich, wo das Konzept bisher überwiegend lediglich akademisch diskutiert wird, siehe http://www.klarsprache.at/ und https://abgb-modernisierung.uni-graz.at/ de/; zu letzterem Projekt vgl. Peter BydlinSKY, Modernisierung des ABGB, in: Österreichische Juristen-Zeitung 70 (2015), S. 869-875. 
schiedlichen Interessenkonstellationen reflektieren, welche in die getroffenen legislativen Entscheidungen eingeflossen sind. Der durch gemeinsame Wurzeln, beispielsweise im römischen Recht, vermittelte Zusammenhalt ist durch immer dynamischer verlaufende Rechtsentwicklung im 2o. Jahrhundert - Carl Schmitt hat schon vor der Jahrhundertmitte von den «steigenden Motorisierungen der Gesetzgebungsmaschine» gesprochen ${ }^{16}$ - weiter zurückgedrängt worden.

\section{NATIONALE RECHTSSPRACHLICHE DIVERGENZ}

So lassen sich beispielsweise heute zumindest sieben deutsche Rechtssprachen nachweisen: die deutsche, die österreichische und die schweizerische ohnedies, dann und darüber hinaus aber auch noch die liechtensteinische, die italienische (oder Südtiroler), die belgische und die luxemburgische. Inwieweit regionale Untergliederungen, wie beispielsweise Bundesländer bzw. Kantone, jeweils noch feinere rechtssprachliche Differenzierungen ausgebildet haben, bleibt vorläufig außer Betracht. Je nach zwischenstaatlicher Konstellation können die nationalen Rechtssprachen zu einander in divergenter oder konvergenter Entwicklung begriffen sein: Die liechtensteinische Rechtssprache beispielsweise war im 19. Jahrhundert durch die Rezeption österreichischen Rechts, beispielsweise des ABGB, geprägt, erfuhr jedoch nach dem Ersten Weltkrieg eine Umorientierung in Richtung auf die schweizerische, insbesondere seit damit begonnen wurde, das liechtensteinische Privatrecht in enger Anlehnung an das schweizerische Zivilgesetzbuch neu zu kodifizieren ${ }^{17}$.

Das österreichische Deutsch im allgemeinen und die österreichische Rechtssprache im besonderen weisen besonders deutlich den Zusammenhang zwischen sprachlicher Divergenz bzw. ihrer Wahrnehmung einerseits und der Entwicklung des politischen Kontexts, vor allem der Selbstwahrnehmung politisch-staatlicher Identität auf. Wenn Max

16 CARL SCHMitT, Die Lage der europäischen Rechtswissenschaft, Tübingen 1943, S. 30.

17 Vgl. Friedrich F.G. KLEINWAEChter, Die neueste Rechtsentwicklung im Fürstentum Liechtenstein, in: Zeitschrift für Schweizerisches Recht NF 42 (1923), S. 356-414, sowie WILHELM BRAUNEDER, Das ABGB in Liechtenstein, in: MiCHAEL GEISTLINGER u.a. (Hg.), 200 Jahre ABGB - Ausstrahlungen. Die Bedeutung der Kodifikation für andere Staaten und andere Rechtskulturen, Wien 2011, S. 61-77, hier S. 76. 
Weinreich eine Sprache als einen Dialekt mit einer Armee und einer Kriegsmarine definiert hat ${ }^{18}$, dann hat er in der Verfügung über eine bewaffnete Macht als eines besonderen Ausdrucks des staatlichen Gewaltmonopols das maßgebliche Zeichen für staatliche Identität erblickt und damit auf den Zusammenhang zwischen Staatsbildung und der Herausbildung einer «nationalen» Sprache, im Falle «plurizentrischer» Sprachen aber eben von mit der staatlichen Ausdifferenzierung einhergehenden nationalen Varietäten hingewiesen ${ }^{19}$.

Zwar ist eine spezifische Lexik des österreichischen Deutsch in seiner konkreten Ausprägung insbesondere auch der in den Kronländern Steiermark und Krain gesprochenen Sprache bereits um 1770 nachhaltig dokumentiert worden, aber gerade diese Dokumentationsleistung ist mit den (nach anfänglichem Zögern letztlich durchgedrungenen) Bestrebungen der maria-theresianischen Schulpolitik zusammengetroffen, das in den österreichischen Kronländern gesprochene Deutsch den sprachlichen Standardisierungsbestrebungen, wie sie von Johann Christoph Gottsched vertreten und theoretisch begründet worden waren, unterzuordnen; ja, mehr noch, das von Johann Siegmund Valentin Popowitsch, Professor der deutschen Sprache und Rhetorik an der Universität Wien, ins Werk gesetzte, aber unvollständig und lange unveröffentlicht gebliebene Dokumentationsprojekt stellt sich geradezu als Manifestation der von ihrem Urheber eingenommenen Gegenposition zu jener Gottscheds dar ${ }^{20}$.

Zur ursprünglichen Ressource (YIVO-bleter 25 [1945], S. 3-18, hier S. 13, online verfügbar unter http://yivolibrarybooks.org/43629) und zur mittlerweile beobachtbaren Beflügelung dieses Wortes vgl. ALEXANDER MAXWELL, When Theory is a Joke: The Weinreich Witticism in Linguistics, in: Beiträge zur Geschichte der Sprachwissenschaft 28 (2018), S. 263-292.

Zum Begriff der «plurizentrischen» Sprache und zur Verknüpfung dieses Konzepts mit der Dynamik der Entwicklung von Staatlichkeit vgl. HeINZ KLOSS, Die Entwicklung neuer germanischer Kultursprachen seit 1800, 2. Aufl., Düsseldorf 1978, S. 66. Das Spektrum politischer Kontexte für die Herausbildung plurizentrischer Sprachen erscheint abgesteckt bei MiCHAEL G. CLYNE, Pluricentric Language/Plurizentrische Sprache, in: UlRich AMmON u.a. (Hg.), Sociolinguistics: An international handbook of the science of language and society/Soziolinguistik: Ein internationales Handbuch zur Wissenschaft von Sprache und Gesellschaft, 2. Aufl., Bd. 1/1, Berlin 2004, S. 296-300, hier S. 296.

Erst 230 Jahre nach Popowitschs Tod ist seine lexikalische Sammlung rekonstruiert und veröffentlicht worden, zugleich ein Ausdruck des mittlerweile erwachten Interesses am österreichischen Deutsch als nationaler Varietät: Johann SiEgmund VALEnTin PoPowiTsCH, Vocabula Austriaca et Stiriaca, hg. von Richard Reutner, 2 Tle., Frankfurt a.M. 2004; zur Person vgl. KuRT FAnIN- 
Österreich verstand sich freilich in unterschiedlichen Ausprägungen bis 1938 als deutscher Staat, zuletzt als der «bessere deutsche Staat», und erst jene tiefe und schmerzliche Zäsur, die durch den «Anschluss» an das nationalsozialistische Deutschland gesetzt oder vielmehr geschlagen worden ist, hat auch eine sprachpolitische Tendenzwende ausgelöst und insbesondere im ersten Jahrzehnt nach 1945, als beispielsweise für einige Jahre anstelle von «Deutsch» ein Schulfach «Unterrichtssprache» geführt worden ist, dazu beigetragen, dass die divergente Entwicklung des österreichischen Deutsch nicht nur bewusster wahrgenommen, sondern sogar gefördert worden ist; seit 1951 war der Besonderheit der österreichischen Lexik ein «Österreichisches Wörterbuch» gewidmet (das freilich der praktischen Verwendung des «Duden» keinen Abbruch getan hat). Langfristig scheint die systematische Förderung des österreichischen Deutsch ein durchaus effektiver Beitrag zur Entwicklung eines österreichischen «Nationalbewusstseins» gewesen zu sein: Den regelmäßig unternommenen demoskopischen Erhebungen zufolge haben 1964 erst $47 \%$ der Befragten der Aussage «Die Österreicher sind eine Nation» zugestimmt, 1993, also knapp vor dem Beitritt zur EU, immerhin bereits 8o \%21.

Mit der Entwicklung des Konzepts der «plurizentrischen Sprachen» ist es auch in der österreichischen Sprachwissenschaft unternommen worden, dieses Konzept zur theoretischen Unterfütterung der Divergenz des österreichischen Deutsch heranzuziehen ${ }^{22}$.

GER, Johann Siegmund Valentin Popowitsch: Ein österreichischer Grammatiker des 18. Jahrhunderts, Frankfurt a.M. 1996; PETER WIESIngER, Johann Siegmund Valentin Popowitsch als Professor für Deutsche Sprache und Wohlredenheit an der Universität Wien 1754-1766, in: Jahrbuch für Internationale Germanistik 47 (2016), S. 45-74.

21 Vgl. ERnSt BRUCKMÜLLER, Nation Österreich: Kulturelles Bewußtsein und gesellschaftlich-politische Prozesse, 2. Aufl., Wien 1996, S. 61ff.

22 Vgl. insb. die zahlreichen Arbeiten von Rudolf Muhr und die von ihm (mit)herausgegebenen Sammelbände wie: Rudolf MuHR (Hg.), Reader: Österreichisches Deutsch kennenlernen, Graz 1993; Rudolf MuHR (Hg.), Internationale Arbeiten zum österreichischen Deutsch und seinen nachbarsprachlichen Bezügen, Wien 1993; Rudolf MuHR/RichaRd SCHROdT/PETER Wiesinger (Hg.), Österreichisches Deutsch: Linguistische, Sozialpsychologische und sprachpolitische Aspekte einer nationalen Variante des Deutschen, Wien 1995; Rudolf MuHR/RichARD SCHRODT (Hg.), Österreichisches Deutsch und andere nationale Varietäten plurizentrischer Sprachen in Europa, Wien 1997; Rudolf MuHR (Hg.), Standardvariationen und Sprachideologien in verschiedenen Sprachkulturen der Welt, Frankfurt a.M. 2005; Rudolf Muhr/Manfred SELlnER (Hg.), 1995-2005: Zehn Jahre Forschung zum Österreichischen Deutsch: Eine Bilanz, Frankfurt a.M. 2006; siehe allg. http://www.oedeutsch.at/. Auf die sprachwissenschaftlichen Kontroversen um das Konzept des österreichischen Deutsch, zum Teil in den erwähnten Sammelwerken wi- 
Als Beleg eignet sich die Rechtssprache in ganz besonderem Ausmaß, zählen doch Rechtstexte ihrer normierten Verbindlichkeit wegen eo ipso zu den für die nationale Varietätenbildung im Kreis plurizentrischer Sprachen besonders signifikanten Textsorten. Und so nimmt es nicht wunder, dass in einem ersten, eingestanden unvollständigen Versuch lexikalischer Herausarbeitung rechtsterminologischer Variation zwischen der österreichischen und der bundesdeutschen Rechtssprache etwa 2000 spezifisch österreichische Rechtsbegriffe identifiziert werden konnten ${ }^{23}$.

Aus den bereits genannten Gründen, insbesondere dem Grund der mit der staatlichen einhergehenden normativen Sonderung, neigt die Rechtssprache selbst dann, wenn nicht staatliche Sprachpolitik divergente Entwicklung der nationalen Varietät zielbewusst fördert, zur konzeptuellen und begrifflichen Spezifizierung. Ja, sie bietet sogar einen besonders griffigen Ansatzpunkt für das «plurizentrische» Modell: wird die normative Dynamik doch von zentralen Legislativorganen vorangetrieben, denen zwar dezentrale Organe der Judikative nachgelagert sind, die sich jedoch im «code law system» an die kodifizierte Terminologie gebunden zeigen. So gibt die Rechtssprache einen viel besseren Beleg für die im Modell erfasste «Plurizentrizität» als beispielsweise die noch variationsreichere Küchensprache, in welcher die regionalen die nationalen Varietäten überlagern ${ }^{24}$.

Die österreichische Rechtssprache weist eine zumindest mehr als 200 Jahre, bis ins Zeitalter der großen zivilrechtlichen Kodifikationen zurückreichende Sonderentwicklung auf ${ }^{25}$, die es leicht macht, in ihr ein selbständiges Zentrum in der plurizentrischen Land-

dergespiegelt, kann und soll an dieser Stelle nicht eingegangen werden; eine - wohl zu oberflächliche - Konzeptualisierung der gegensätzlichen Positionen versucht JАKOB EBNER, Duden: Wie sagt man in Österreich? Wörterbuch des österreichischen Deutsch, 4. Aufl., Berlin 2014, S. 440 .

Rudolf Muhr/Marlene PeInhopf, Wörterbuch rechtsterminologischer Unterschiede Österreich-Deutschland, Frankfurt a.M. 2015. Vgl. etwa Heinz DieTER PoHL, Die österreichische Küchensprache: Ein Lexikon der typisch österreichischen kulinarischen Besonderheiten, Wien 2007.

Eine anhand eines Seitenstranges der österreichischen Rechtsentwicklung, nämlich am Beispiel der Vorschriften des Studienrechts seit dem späten 18. Jahrhundert unternommene diachrone Analyse der Syntax, Stilistik und Lexik der österreichischen Rechtssprache bietet PAUL RösSLER, 
schaft der deutschen Rechtssprachen repräsentiert zu sehen: Mit dem Allgemeinen bürgerlichen Gesetzbuch von 1811, das seinerseits auf das Westgalizische Gesetzbuch von 1797 zurückgeht, ist auch ein seither beständig weitergeführter und weiterentwickelter Kernbestand österreichischer Rechtsterminologie kodifiziert worden, und auch wenn sich das ABGB mit seinen Teilnovellen der Jahre 1914-16, auch dies durch den historischpolitischen Kontext bedingt, an das BGB angenähert hat, so ist ihm doch weiterhin größere Eigenständigkeit diesem gegenüber konzediert worden als dem Schweizer ZGB26. Und so hat für einen Ausschnitt des bürgerlichen Rechts, nämlich das Erb- und Familienrecht, eine exemplarische Studie nicht weniger als 96 terminologische - zum Teil auch konzeptuelle - Austriazismen herausgearbeitet ${ }^{27}$.

Eine noch größere Vielfalt als die deutschen weisen naturgemäß die englischen, französischen, spanischen, portugiesischen oder arabischen Rechtssprachen auf. Angesichts solcher Vielfältigkeit nimmt es nicht wunder, dass schon äquivalente Transformationen zwischen Rechtstexten, die jeweils einem solchen Kreis der ein und derselben «Hintergrundsprache» angehörenden Rechtssprachen zuzurechnen sind, auf oft nahezu unüberwindliche Schwierigkeiten stoßen. Nicht minder heikel erscheinen äquivalente Transformationen von Rechtstexten in «alltagssprachliche» Texte derselben nationalen Sprache bzw. Varietät: Selbst in jenen seltenen Fällen, in denen sich die Rechtssprache injektiv auf die Alltagssprache abbilden lässt, mag der entsprechende alltagssprachliche Begriff anders konnotiert sein als der rechtssprachliche («Studiengebühren» vs. «Studienbeiträge» aus der österreichischen Alltags- bzw. Rechtssprache gibt ein anschauliches Beispiel). Viel häufiger aber ist eine äquivalente Transformation aus der Rechts- in die Alltagssprache nur über Umschreibungen möglich, weil die Alltagssprache nicht über «maßgeschneiderte» Begriffe verfügt, um die normativen Konzepte auszudrücken.

Entwicklungstendenzen der österreichischen Rechtssprache seit dem ausgehenden 18. Jahrhundert, Frankfurt a.M. 1994.

26 So von ERnSt A. KRAmer, Der Einfluß des BGB auf das schweizerische und österreichische Privatrecht, in: Archiv für die civilistische Praxis 200 (2000), S. 365-400.

27 Michael KuCHARSKI, Austriazismen im Erb- und Familienrecht, Diplomarbeit, Wien 2009. 


\section{DIE SPRACHE(N) DES EUROPÄISCHEN RECHTS}

Und nun kommt noch das «Mehrebenensystem» hinzu, wie es sich in Europa als die Überlagerung verschiedener normativer Systeme manifestiert, die zueinander teilweise im Verhältnis der Verdrängung, teilweise der Koexistenz, teilweise aber auch der Koevolution stehen. Eine kohärente «europäische» Rechtssprache hat sich noch gar nicht herausgebildet, vollzieht sich doch die europäische Rechtsetzung unter sprachmedialer Vermittlung durch Fragmente konzeptuell so unterschiedlicher Rechtssprachen wie insbesondere der englischen und der französischen ${ }^{28}$. Es entstehen rechtssprachliche schwimmende Inseln, die den breiten Strom des europäischen normativen Prozesses hinuntertreiben. Dieser Strom fließt naturgemäß nicht in einem regulierten Bett, sondern verzweigt sich nach jeder Engstelle - mit dieser Metapher könnte ein bestimmter europäischer Rechtsetzungsvorgang beschrieben werden - in ein Gewirr von Nebenarmen: mitgliedstaatlichen Rechtsetzungsprozessen, mit welchen umsetzungsbedürftige europäische Rechtsakte in nationales Recht transformiert (oder nicht umsetzungsbedürftige zumindest nicht selten, beispielsweise durch Erlassung nationaler Organisationsvorschriften, begleitet) werden; für solche Transformationen ist substantielle Äquivalenz - bei vordefinierten Spielräumen für mögliche Divergenz - vorgeschrieben, was die nationale Umsetzung europäischer Rechtsakte zu einer rechtspolitisch schwierigen Gratwanderung zwischen Konzeptualisierung und Formulierung macht.

Liegen die europäischen Rechtsakte auch in grundsätzlich gleichermaßen authentischen Sprachfassungen in allen Amtssprachen vor, so belegt die praktische Erfahrung die Schwierigkeit, wenn nicht die Unmöglichkeit vollständiger äquivalenter Transformation von Rechtstexten. Der deklaratorische Anspruch des EuGH zielt daher auf einen

Vgl. ISOLDE BuRR/Friedrich MÜlLeR (Hg.), Rechtssprache Europas: Reflexion der Praxis von Sprache und Mehrsprachigkeit im supranationalen Recht, Berlin 2004; ANNE LISE KJÆR, Legal Translation in the European Union: A Research Field in Need of a New Approach, in: KRZYSZTOF KREDENS/STANISLAW GozDZ-RoszKowsKi (Hg.), Language and the Law: International Outlooks, Frankfurt a.M. 2007, S. 69-95; ISOLDE BURR, Linguistische Aspekte zu authentischen mehrsprachigen Rechtstexten: Erstvotum zum Beitrag von Werner Hauck, in: Aktuelle Juristische Praxis 18 (2009), S. 750-760. Zur translatorischen Praxis vgl. Cliff RoBERTSON, EU Legislative Texts and Translation, in: Le ChEnG/King Kui Sin/Anne WAGnER (Hg.), The Ashgate Handbook of Legal Translation, Farnham 2014, S. 155-175. 
Vergleich aller Sprachfassungen, um aus deren semantischer Schnittmenge so etwas wie den Telos des Rechtsaktes ableiten zu können ${ }^{29}$. Eingelöst werden kann dieser Anspruch naturgemäß kaum, weder praktisch (angesichts der sich durch einen solchen Vergleich ja nur potenzierenden Problematik sprachlicher Transformation) noch theoretisch (würde darin doch so etwas wie ein stochastisches Modell normativer Interpretationsrichtigkeit vorausgesetzt sein, dem es zumindest bisher an einer überzeugenden Begründung mangelt).

Aus dieser Erfahrung hat der EuGH, wenn auch uneingestanden, die im Prinzip gleiche Schlussfolgerung gezogen wie, eingestanden, der altösterreichische Reichsgesetzgeber, der vor einem analogen Problem gestanden ist: der Veröffentlichung der Reichsgesetze in den (damals acht) Sprachfassungen des Reichsgesetzblattes - gemäß Art. 19 des Staatsgrundgesetzes über die allgemeinen Rechte der Staatsbürger ${ }^{30}$ galten alle landesüblichen Sprachen als gleichberechtigt - und der sich aus inäquivalenten sprachlichen Transformationen ergebenden Aporie, aus welcher nur durch Bevorzugung der «Produktionssprache» des jeweiligen Rechtsaktes - und das war im alten Österreich die deutsche, der durch $§ 2$ des Reichsgesetzblattgesetzes ${ }^{31}$ der Authentizitätsvorrang zuerkannt war - ausgebrochen werden konnte. Der Rekurs auf die «Produktionssprache», in welcher der Rechtsakt ursprünglich konzipiert und gutteils auch der rechtspolitische Diskurs über den zu erlassenden Rechtsakt geführt worden ist, gewährleistet am ehesten die Rückbindung an den in ihm zum Ausdruck gebrachten rechtspolitischen Willen und damit die ihm innewohnende politische Legitimation. In der europäischen Rechtsetzung ist das heute in der Regel die englische oder die französische Sprache; dabei tritt in der Verfahrenspraxis des EuGH aufgrund seiner eigenen Arbeitssprache eine gewisse Präferenz für die französische Version zutage ${ }^{32}$. Die Lehre, nach dem Grundsatz,

29 Siehe insb. EuGH, Rs. C-188/03. Hinweise auf ältere Urteile gibt KJÆR, wie Anm. 28, S. 88. Vgl. auch KARIN LUTTERMANN, Mehrsprachigkeit am Europäischen Gerichtshof: Das Referenzsprachenmodell für ein europäisches Sprachenrecht, in: DorotheE Heller/KonRAd EHRLICH (Hg.), Studien zur Rechtskommunikation, Bern 2007, S. 47-80.

30 RGBl. Nr. 142/1867. Vgl. dazu das Standardwerk von GERALD STOURZH, Die Gleichberechtigung der Nationalitäten in der Verfassung und Verwaltung Österreichs 1848-1918, Wien 1985.

31 RGBl. Nr. 113/1869.

32 Vgl. NinON COLNERIC, Auslegung des Gemeinschaftsrechts und gemeinschaftsrechtskonforme Auslegung, in: ZEuP 12 (2005), S. 225-233. 
dass nicht sein könne, was nicht sein dürfe, leugnet freilich solche Präferenzen überwiegend 33 .

Die praktische Schwierigkeit, die substantielle normative Äquivalenz nicht nur an unterschiedlichen Sprachfassungen desselben europäischen Rechtsaktes, sondern umso mehr an nationalen Umsetzungsakten zu erkennen, ist gewiss nicht die einzige, aber eine Motivation gewesen, die den EuGH in der Ausbildung seiner «richtliniennahe» Umsetzung fordernden Judikatur geleitet hat ${ }^{34}$. «Richtliniennah» ist sehr unmittelbar sprachgebunden zu verstehen: Am «richtliniennächsten» in diesem Sinn ist ein «copy and paste»-Verfahren, welches sprachliche Transformation durch sprachlichen Transfer ersetzt; das «Ziel» des umsetzungsbedürftigen europäischen Rechtsaktes umfasst in dieser Perspektive immer mehr auch seinen Wortlaut ${ }^{35}$. Im Blick auf die zuvor festgestellte Unbestimmtheit aber bereits der sprachlichen Transformation des europäischen Rechtsaktes aus der «Produktionssprache» in die jeweilige nationale Amtssprache gewährleistet freilich nicht einmal dieses Verfahren die sichere normative Äquivalenz, sodass die Referenz auf die produktionssprachliche Fassung des europäischen Rechtsaktes im nationalen Umsetzungsprozess jedenfalls nicht unterbleiben darf.

Mehr noch als im nationalen Rechtsetzungsverfahren gewinnen im europäischen, und in der Transformation seiner Ergebnisse, datenbankgestützte Hilfsmittel an Bedeutung, welche die vom individuellen Intellekt nicht mehr beherrschbare terminologische Fülle

33 So etwa Rüdiger STOTZ in Karl RieSEnHuber (Hg.), Europäische Methodenlehre: Handbuch für Ausbildung und Praxis, Berlin 2006, S. 538; a.A. Karl RieSEnhuber/Stefan Grundmann, Die Auslegung des Europäischen Privat- und Schuldvertragsrechts, in: Juristische Schulung 41 (2001), S. 529-536, hier S. $529 f$.

Vgl. JÜRGEN BAST, Handlungsformen und Rechtsschutz, in: ARMIN VON BOGDANDY/JÜRGEN BAST (Hg.), Europäisches Verfassungsrecht: Theoretische und dogmatische Grundzüge, 2. Aufl., Berlin 2009, S. 489-557, hier S. 504f.

Vgl. aus der Sicht der österreichischen Legistik JoHANnEs STABENTHEINER, Probleme bei der Umsetzung zivilrechtlicher EU-Richtlinien am Beispiel der Time-Sharing-Richtlinie, in: Juristische Blätter 119 (1997), S. 65ff.; DERS., Legistik im europäischen Arbeitsfeld: Herausforderungen an die Zivillegistik bei der Schaffung europäischen Rechts und seiner innerstaatlichen Umsetzung, in: Österreichische Juristen-Zeitung 65 (2010), S. 263-268. 
auffangen, erschließen und für die sprachliche Transformation aufbereiten sollen ${ }^{36}$. Solche Terminologiedatenbanken, ihnen voran die von den Übersetzungsdiensten der EU entwickelte und verwendete Datenbank IATE (Inter-Active Terminology for Europe), entstehen durch annotierende Termextraktion aus den Texten und wirken durch ihre Verfügbarkeit auf den Prozess der Textproduktion zurück. Mögen sie heute auch noch recht flache Strukturen aufweisen und so die Komplexität semantischer Beziehungen nur sehr unzureichend abbilden, so bildet doch allein die Menge gesammelter Information einen wichtigen Grundstock, auf welchem künftige Ontologien des europäischen Rechtssystems aufsetzen können.

\section{E. RECHTSSPRACHE(N) ZWISCHEN KONVERGENZ UND DIVERGENZ}

Unter den Ängsten, die es in Österreich im Vorfeld des Beitritts zur Europäischen Union - bzw. den Europäischen Gemeinschaften, wie sie in den Jahren der Beitrittsverhandlungen und des nationalen Beitrittsdiskurses noch hießen - zu überwinden galt, war auch die Angst vor einem Verlust an sprachlicher Identität. Um dieser Angst zumindest ein Zeichen entgegenzusetzen, legte Österreich Wert darauf, in das umfangreiche Vertragswerk über den Beitritt auch ein Protokoll aufgenommen zu sehen, welches die Verwendung der darin festgelegten, vom bundesdeutschen Sprachgebrauch abweichenden Begriffe des österreichischen Deutsch in europäischen Rechtsakten gewährleisten sollte ${ }^{37}$. Dieses kurz sogenannte «Austriazismen-Protokoll» be-

36 Vgl. dazu grundsätzlich GERHARD Budin, Wissensorganisation und Terminologie: Die Komplexität und Dynamik wissenschaftlicher Informations- und Kommunikationsprozesse, Tübingen 1996.

37 Protokoll Nr. 10 über die Verwendung spezifisch österreichischer Ausdrücke der deutschen Sprache im Rahmen der Europäischen Union zum EU-Beitrittsvertrag, BGBl. Nr. 45/1995 (bzw. CELEX:11994N/PRO/10). Siehe dazu die Regierungsvorlage betr. den EU-Beitrittsvertrag, 11 d.B. zu den Sten.Prot. des Nationalrates, XIX. GP, S. 430, und den Bericht des Außenpolitischen Ausschusses des Nationalrates über diese Regierungsvorlage, 25 d.B. zu den Sten.Prot. des Nationalrates, XIX. GP, S. 8. Vgl. auch FABIAN LUTZ, Das Austriazismenprotokoll im EU-Beitrittsvertrag, in: ecolex 1994, S. 880-883. 
nennt 23 Begriffe, durchwegs Sachbegriffe aus dem Lebensmittelrecht, keine Rechtsbegriffe, d.h. keine Begriffe für Rechtsinstitute ${ }^{38}$, und ist zwiespältig beurteilt worden. Auf der einen Seite konnte in der Beitrittskampagne propagandistisch verkündet werden, «Erdäpfelsalat» bleibe «Erdäpfelsalat» ${ }^{39}$, und damit war der unmittelbare praktische Zweck schon erreicht: Wenn die österreichischen Wählerinnen und Wähler nicht mehr befürchten mussten, künftig «Kartoffeln» anstelle von «Erdäpfeln» oder «grüne Bohnen» statt «Fisolen» (die im Bundesland Kärnten im Übrigen als «Strankalan» bekannt sind) kaufen (und essen) zu müssen, erschien eine ihrer Europaängste ausgeräumt; auch Identität geht durch den Magen. Gleichzeitig konnten die akademischen Vertreter des Konzepts eines österreichischen Deutsch befriedigt feststellen, dass mit dem Protokoll die rechtlich relevante sprachliche Eigenständigkeit des österreichischen Deutsch grundsätzlich anerkannt worden war ${ }^{40}$.

Auf der anderen Seite gaben Kritiker des Protokolls in seiner konkreten Ausformung zu bedenken, dass damit das Ausmaß dieser sprachlichen Eigenständigkeit eben auf die darin enthaltenen 23 Begriffe beschränkt erscheinen, also daraus ein Umkehrschluss auf eine erschöpfende Aufzählung der dem österreichischen Deutsch eigentümlichen Begriffe von rechtlicher Relevanz gezogen werden könnte und somit eben gerade nicht

38 Ein von einer österreichischen Praktikantin beim Übersetzungsdienst der Kommission erarbeitetes Glossar verwaltungsrechtlicher «Austriazismen» bleib in den Beitrittsverhandlungen unberücksichtigt, was diplomatiesoziologisch auch damit zu erklären ist, dass das Protokoll österreichischerseits von Beamten der Bundesministerien für Land- und Forstwirtschaft bzw. Gesundheit, Sport und Konsumentenschutz verhandelt wurde, die sich auf die lebensmittelrechtliche Terminologie konzentrierten. Das erwähnte Glossar ist immerhin später in überarbeiteter und erweiterter Form publiziert worden und bildet somit einen nützlichen, aber unverbindlichen Arbeitsbehelf: HEIDEMARIE MARKHARDT, Wörterbuch der österreichischen Rechts-, Wirtschafts- und Verwaltungsterminologie, 2. Aufl., Frankfurt a.M. 2010.

Die Eindrücklichkeit dieses - von der Wiener SPÖ plakatierten - Slogans mag schon dadurch belegt erscheinen, dass der populäre «Austropop»-Sänger Georg Danzer ein selbstironisch die österreichische Neigung zu beharrlicher Nabelbeschau karikierendes Lied unter diesem Titel in sein 1995 erschienenes Album «Große Dinge» aufgenommen hat; siehe http://www.georgdanzer.at/lieder/erdaepfelsalat-bleibt-erdaepfelsalat/.

So etwa RudolF DE CILLIA, Erdäpfelsalat bleibt Erdäpfelsalat: Österreichisches Deutsch und EUBeitritt, in: Rudolf Muhr/Richard Schrodt/Peter Wiesinger (Hg.), Österreichisches Deutsch: Linguistische, Sozialpsychologische und sprachpolitische Aspekte einer nationalen Variante des Deutschen, Wien 1995, S. 122-132, hier S. 130. 
der weit ausgreifenden Besonderheit der österreichischen Varietät des Deutschen Rechnung getragen wäre. Und auch wenn sich die EU sowohl in Art. 22 der Grundrechtscharta als auch in Art. 3 Abs. 3 EUV zum Prinzip der Sprachenvielfalt als Grundwert bekennt, ist ihrer Rechtsordnung, mit dem «Austriazismen-Protokoll» als einer Ausnahme ${ }^{41}$, das Konzept der nationalen Sprachvarietäten fremd geblieben.

In der Praxis war zum Zeitpunkt des Beitritts der Republik Österreich zur Europäischen Union am 1. Jänner 1995 die europäische Rechtssprache in der deutschen Fassung der europäischen Rechtsakte naturgemäß von der bundesdeutschen Rechtssprache geprägt. Als eines der Gründungsmitglieder der EWG und der anderen Europäischen Gemeinschaften hatte nicht nur die Bundesrepublik Deutschland als Staat, als Vertragsund Verhandlungspartner die europäische Rechtsentwicklung maßgeblich beeinflusst, sondern die Dolmetsch- und Übersetzungsdienste der europäischen Institutionen hatten für die Übersetzung der europäischen Rechtsakte und der anderen rechtlich relevanten Dokumente ins Deutsche vier Jahrzehnte lang überwiegend (bundes)deutsche Fachkräfte herangezogen. Dementsprechend waren auch die Übersetzungshilfsmittel, insbesondere die damalige, seit den 1970er Jahren geführte Terminologiedatenbank «Eurodicautom» - aus welcher IATE hervorgegangen ist - mit bundesdeutscher Rechtsterminologie gespeist worden. Natürlich wurden die Reihen der Übersetzungsdienste von 1995 an auch aus österreichischem Fachpersonal ergänzt, aber aus dem üblichen Laufbahnverlauf musste sich bis zu dessen Aufrücken in höherrangige und einflussreichere Supervisorenpositionen eine Verzögerung ergeben.

Immerhin - eine Bresche war geschlagen, rechtlich durch das «Austriazismen-Protokoll», praktisch durch die österreichische Vertretung in den Übersetzungsdiensten, und nicht zuletzt durch geschicktes «Netzwerken» ist es mittelfristig gelungen, auch IATE mehr und mehr um Begriffe des österreichischen Deutsch anzureichern. Das kann naturgemäß nichts daran ändern, dass eben nur eine verbindliche deutsche Sprachfassung der europäischen Rechtsakte existiert, und deren terminologische Gestaltung spiegelt, wenn nicht Neologismen gebildet werden, im Zweifel naturgemäß eher die bundesdeutsche als die österreichische rechtssprachliche Tradition wider.

41 Zur Anwendungspraxis siehe https://ec.europa.eu/info/sites/info/files/austrian_expressions_de.pdf. 
Die Neologismenbildung erscheint auf den ersten Blick als willkommener Ausweg nicht nur aus der Aporie der Kontraposition zweier nationaler Varietäten einer Sprache, sondern auch aus der Gefahr, europäische Rechtsbegriffe unwillkürlich vor dem Hintergrund der je eigenen nationalen Rechtsterminologie zu interpretieren und ihnen damit unter Umständen eine Bedeutung zu hinterlegen, die ihnen vor dem Hintergrund eines kompromisshaft zu einem Ergebnis geführten, verschiedene nationale Rechtstraditionen amalgamierenden oder transzendierenden europäischen Rechtsetzungsvorganges gar nicht zukommt. Freilich wirken solche Neologismen manchmal allzu willkürlich und, wenn beispielsweise in der deutschen Fassung der DSGVO der bisherige «Auftraggeber» der DSR «Verantwortlicher», der bisherige «Dienstleister» aber «Auftragsverarbeiter» heißt, erschließt sich dem naiven Konsumenten des Rechtstextes die Rationalität dieser terminologischen Dynamik zunächst nicht, es sei denn, sie will lediglich zum Ausdruck bringen, dass nun alles anders sei.

Unmittelbar anwendbare europäische Rechtsakte ragen solcherart auch terminologisch in gewachsene nationale Rechtsordnungen zunächst als erratische Blöcke hinein. In manchen Fällen, wie in jenem der DSGVO, muss die nationale Rechtsordnung durch Anpassungsgesetzgebung auch terminologisch kompatibel gemacht werden. Anders stellt sich die Problematik der nationalen Transposition nicht unmittelbar anwendbarer Rechtsakte dar: Sie würde grundsätzlich, sofern die nationale Rechtsordnung äquivalente Konzepte kennt, die terminologische Transformation in die vertraute nationale Rechtssprache erlauben, wenn ihr nicht das Postulat der «richtliniennahen» Umsetzung im Weg stünde. Das EU-Addendum zu den österreichischen Legistischen Richtlinien hat kurz nach dem Beitritt zur Europäischen Union bei der Richtlinienumsetzung der Verwendung der «österreichischen Gesetzessprache» den Vorzug vor dem Transfer systemfremder Terminologie eingeräumt ${ }^{42}$. Realität aber ist, was der vormalige Präsident des österreichischen Verfassungsgerichtshofes, Karl Korinek, kritisch «Xerox-Gesetzgebung» genannt hat, die wortnahe Umsetzung der europäischen Rechtsakte in Gestalt nationaler Sondergesetze außerhalb vorhandener Kodifikationen, also die nicht

42 Z 35 des EU-Addendum zu den LegRL 1990, https://www.justiz.gv.at/file/2c94848a60c 158380160e4e3747c0c33.de.0/addendum.doc. Dazu kritisch DoRIS LIEBWALD, Europäische Rechtsbegriffe und österreichische Rechtssprache: Die Transformation von EU-Richtlinien in nationales Recht, in: Journal für Rechtspolitik 21 (2013), S. 294-310. 
nur terminologische, sondern auch systematische Störung des gewachsenen Gefüges der nationalen Rechtsordnung. Hinzu kommt natürlich die tendenziell voranschreitende Einschränkung des nationalen Umsetzungsspielraumes durch das zum Regelfall werdende Konzept der Vollharmonisierung43.

Ist die damit einhergehende wachsende Divergenz innerhalb der Systematik der nationalen Rechtsordnung und der Terminologie der nationalen Rechtsprache der Preis, den wir für die schrittweise Konvergenz zu einer europäischen Rechtsordnung (und Rechtssprache) zu zahlen bereit sein müssen? Der Weg dahin ist jedenfalls noch weit, und im Hier und Heute sehen wir uns mit Inkohärenz konfrontiert: wachsender Inkohärenz in den nationalen Rechtssystemen, aber auch Inkohärenz der europäischen Rechtsordnung, bedingt nicht zuletzt durch die Unebenheiten einer unüberschaubar gewordenen europäischen rechtsterminologischen Landschaft. Allein der öffentlich zugängliche Teil von IATE umfasst mehr als eine Million multilinguale Einträge mit nahezu acht Millionen Begriffen ${ }^{44}$ !

Die Zukunft Europas, die vor einem Vierteljahrhundert, als die Republik Österreich ihre Beitrittsentscheidung getroffen hat, wie ein gerader Weg zu vertiefter Integration vorgezeichnet erschienen ist, wirkt heute in Frage gestellt. Auf der einen Seite scheint die große integrationspolitische Vision zu fehlen und sich die Europäische Union politischadministrativ in oftmals unzulänglichem Krisenmanagement zu erschöpfen, auf der anderen Seite treten Diskurse um die (Re-)Konstruktion nationaler Identitäten in Konkurrenz mit dem Diskurs um die Konstruktion einer übergreifenden europäischen Identität.

Ein Medium eines Identitätsdiskurses ist das Recht: So war, um ein Beispiel für die Funktion des Rechts in der Konstruktion nationaler Identität zu benennen, das Rechts-

43 Vgl. AnNE-Christin MitTwoch, Vollharmonisierung und Europäisches Privatrecht: Methode, Implikationen und Durchführung, Berlin 2013.

44 https://iate.europa.eu/home, Stand 15. November 2019, nennt 1015409 Einträge und 7993 143 Begriffe. Die Zahl der Einträge, die schon bei über 1,4 Millionen gelegen ist, ist durch die automationsunterstützte Bereinigungsarbeit immerhin signifikant zurückgegangen; vgl. PAULA ZORRILLA-AGUT, When IATE met LISE: LISE clean-up and consolidation tools take on the IATE challenge, in: GERHARD Budin/VESNA LuŠICKY (Hg.), Languages for Special Purposes in a Multilingual, Transcultural World: Proceedings of the 19th European Symposium on Languages for Special Purposes, 8-10 July 2013, Vienna, Austria, Wien 2014, S. 536-545. 
Überleitungsgesetz vom 1. Mai $1945^{45}$, einer der ersten Rechtsakte der nach Wiedererlangung der staatlichen Unabhängigkeit gebildeten provisorischen österreichischen Staatsregierung, Ausdruck und Mittel nicht nur der Reinigung der österreichischen Rechtsordnung, sondern auch des österreichischen Selbstverständnisses von den Spuren des «Anschlusses», des Verlustes der nationalen Selbstständigkeit und der sieben Jahre währenden nationalsozialistischen Gewaltherrschaft ${ }^{46}$. Und wenn beispielsweise ein Rundschreiben des österreichischen Bundeskanzleramtes vom 19. März 1948 den Bundesministerien empfahl, in ihrer legistischen Arbeit Wortbildungen aus den Jahren 1938 bis 1945, die der österreichischen «Mundart» fremd wären - das linguistische Konzept der nationalen Sprachvarietät stand eben noch nicht zur Verfügung -, zu vermeiden ${ }^{47}$, dann wurde gezielt die Rechtsprache instrumentalisiert, um den österreichischen Identitätsdiskurs voranzutreiben.

Nicht minder ist das Recht ein Angelpunkt des europäischen Identitätsdiskurses: die Europäische Union - bzw. schon die EWG - versteht sich (in einem auf Walter Hallstein zurückgeführten Begriff) als «Rechtsgemeinschaft» 48 und das Recht als das Mittel zur

Österreichische Juristen-Zeitung 1 (1946), S. 3-5; weiters LEOPOLD WERNER, Das Österreich vom 13. März 1938 und vom 27. April 1945: Eine Untersuchung über das Problem der Kontinuität oder Diskontinuität der österreichischen Rechtsordnung, in: Juristische Blätter 68 (1946), S. 2-7; DERS., Das Wiedererstehen Österreichs als Rechtsproblem, in: Juristische Blätter 68 (1946), S. 85-93, 105-108, Juristische Blätter 69 (1947), S. 137-145, 161-164; heute wird das Ergebnis der Rechtsüberleitung retrospektiv kritischer gesehen, vgl. etwa JOACHIM STERN, «...nicht für die Rechtsbereinigung geeignet», in: juridikum 2000, S. 197-201; ILSE REITERZATLOUKAL/MARIA SAGMEISTER, Die Rechtsüberleitung 1945 und die Kontinuität nationalsozialistischen Rechts, in: juridikum 2015, S. 188-198.

Siehe EuGH, Rs. 294/83, Rn. 23; Rs. 314/85, Rn. 16; Rs. C-314/91, Rn. 8, etc. Aus der Fülle der Literatur vgl. etwa MANFRED ZULEEG, Die Europäische Gemeinschaft als Rechtsgemeinschaft, in: Neue Juristische Wochenschrift 47 (1994), S. 545-549; WolfGANG BlomEYER/KarL AlBREChT SCHACHTSCHNEIDER (Hg.), Die Europäische Union als Rechtsgemeinschaft, Berlin 1995; INGOLF PERNICE, Begründung und Konsolidierung der Europäischen Gemeinschaft als Rechtsgemeinschaft, in: MANFRED ZuleEg (Hg.), Der Beitrag Walter Hallsteins zur Zukunft Europas, BadenBaden 2003, S. 56-70; FRANZ C. MAYER, Europa als Rechtsgemeinschaft, in: GUNNAR FoLKE SCHUPPERT/INGOLF PERNICE/UlRICH HALTERN (Hg.), Europawissenschaft, Baden-Baden 2005, S. 429-487; ROBERT ULLERICH, Rechtsstaat und Rechtsgemeinschaft im Europarecht: Eine dogmatische und terminologische Untersuchung der europäischen Verträge und der Rechtspre- 
Operationalisierung jener Grundwerte, zu denen sie sich bekennt, in den Fokus des Diskurses zu rücken, wird umso wichtiger in dem Maße, in welchem andere integrationsleitende Motive, wie etwa das ökonomische, an Attraktivität verlieren und die politische Problemlösungskapazität der Europäischen Union abzunehmen scheint, was dem EuGH und seiner Rechtsprechung in Verfolgung der Ziele des europäischen Primärrechts eine zentrale Rolle in der Weiterentwicklung der Integration eingeräumt hat und dem alten Wort von einem «Europa der zwei Geschwindigkeiten» einen neuen Sinn verleiht ${ }^{49}$.

Wird das europäische Recht zum einen wirkmächtig genug sein, die Stockungen im europäischen Integrationsprozess überwinden zu helfen und über die institutionelle hinaus auch eine mentale europäische Integration wachsen zu lassen? Wird zum anderen die europäische Rechtsentwicklung einen kohärenten europäischen Rechtsraum, ein europäisches Rechtsbewusstsein als Ausdruck der vielberufenen europäischen Grundwerte und nicht zuletzt eine europäische Rechtswissenschaft entstehen lassen, um so jene Fragmentierung der Jurisprudenz zu überwinden, die im Zeitalter der Entstehung der Nationalstaaten Rudolf von Ihering beklagt hat? Hat doch der große deutsche Rechtsgelehrte um die Mitte des 19. Jahrhunderts gemeint: «... die Wissenschaft ist zur Landesjurisprudenz degradirt, die wissenschaftlichen Gränzen fallen in der Jurisprudenz mit den politischen zusammen. Eine demüthigende, unwürdige Form für eine Wissenschaft!»50

chung des Europäischen Gerichtshofs - zugleich eine Gegenüberstellung zum Bundesverfassungsrecht, Baden-Baden 2011; ULRIKE SCHUSTER, Das Kohärenzprinzip in der Europäischen Union, Baden-Baden 2017, S. 155f.; den identitären Gesichtspunkt des Konzepts der EU als «Rechtsgemeinschaft» kritisiert jüngst ULRIKE GUÉROT, Europäische Republik: Von der EURechtsgemeinschaft zur europäischen Demokratie?, in: juridikum 2018, S. 489-497.

49 Vgl. beispielsweise SuSANNE SCHMIDT, Europäische Integration zwischen judikativer und legislativer Politik, in: MARTIn HÖPNER/ARMin SCHÄFER (Hg.), Die Politische Ökonomie der europäischen Integration, Frankfurt a.M. 2008, S. 101-127; MARCUS HöRETH, Die Selbstautorisierung des Agenten: Der Europäische Gerichtshof im Vergleich zum U.S. Supreme Court, Baden-Baden 2008; MARTIN HÖPNER, Der Europäische Gerichtshof als Motor der Integration: Eine akteursbezogene Erklärung, in: Berliner Journal für Soziologie 21 (2011), S. 203-229.

50 RUdolF VON IHERING, Geist des römischen Rechts auf den verschiedenen Stufen seiner Entwicklung, 2. Aufl., 1. Tl., Leipzig 1866, S. 15; vgl. aus der Sicht der Rechtsvergleichung dazu FILIPPO RANIERI, Die Rechtsvergleichung und das deutsche Zivilrecht im 20. Jahrhundert: Eine wissenschaftshistorische Skizze, in: HARTMUT KAELBLE/JÜRGEN SCHRIEWER (Hg.),Vergleich und Transfer: 
Vermag das Unionsrecht, vergleichbar dereinst dem römischen Recht, als Anknüpfungspunkt für die Rekonstruktion einer europäischen Jurisprudenz zu dienen? Wird es eine jener des römischen Rechts vergleichbare kohärente und zugleich multilinguale Rechtsterminologie entwickeln? Heute trägt das Unionsrecht in seiner sektoralen Überlagerung nationaler Rechtsordnungen und in seiner Förderung ihrer systematischen und terminologischen Divergenz mehr zum Entstehen europäischer Rechtsunordnung bei, aber gerade die Jurisprudenz erscheint aufgerufen, die Politik als Rechtspolitik daran zu erinnern, was Europa als «Rechtsgemeinschaft» bedeuten kann und soll.

\section{F. AUFGABEN DER RECHTSINFORMATIK}

«Vor dem Gesetz steht ein Türhüter», erfahren wir von Kafka. Mehr noch: «Von Saal zu Saal stehen aber Türhüter, einer mächtiger als der andere.» Und obgleich Kafkas «Mann vom Lande» denkt, «das Gesetz soll doch jedem und immer zugänglich sein», vermag er in Kafkas Parabel «Vor dem Gesetz» schon den ersten dieser Türhüter schlussendlich nicht zu überwinden ${ }^{51}$.

«Zugang zum Recht» kann Verschiedenes benennen: den Zugang zum Text einer Rechtsvorschrift - oder den Zugang zu seiner «Bedeutung» bzw. zu deren «Verständnis». Oder aber natürlich auch, in einem rechtssoziologischen Sinn, den Zugang zur Anwendung, zur Durchsetzung des Rechts, zu den Institutionen des Rechtssystems.

Einer der mächtigsten jener Türhüter, die den Zugang zum Recht verwehren oder zumindest erschweren, ist die Rechtssprache. Wird der formale Zugang zu den Rechtstexten von der Rechtsinformatik heute in einer Weise unterstützt, die allen, welche noch mit Loseblattausgaben aufgewachsen sind, unverminderte Dankbarkeit abnötigt, so

Komparatistik in den Sozial-, Geschichts- und Kulturwissenschaften, Frankfurt a.M. 2003, S. 221-250, hier S. 234f.; THOMAS HENNINGER, Europäisches Privatrecht und Methode: Entwurf einer rechtsvergleichend gewonnenen juristischen Methodenlehre, Tübingen 2009, S. 31; jüngst Tim PHILIPP HoLLER, «Ist die Jurisprudenz eine Wissenschaft?» Zum 150-jährigen Jubiläum von Jherings Wiener Antrittsvorlesung am 16. Oktober 2018, in: ZJS 2018, S. 503-513, hier S. 506.

Text: https://de.wikisource.org/wiki/Vor_dem_Gesetz. 
bleibt die Semantik der Rechtsvorschriften auch für die Rechtsinformatik eine Herausforderung. Erich Schweighofer hat sich dieser Herausforderung in vielen Projekten und wissenschaftlichen Arbeiten gestellt, insbesondere im Zusammenhang mit der Konzeptualisierung des Rechts bzw. der Rechtssprache in Thesauri und Ontologien als Ressourcen für die automationsunterstützte semantische Verarbeitung von Rechtstexten ${ }^{52}$.

Ein kurzer abschließender Blick auf Aufgabenstellungen, welche die Rechtssprache der Rechtsinformatik eröffnet, zeigt ihre Vielfalt und ihr Potential; dabei handelt es sich nicht nur um analytische, sondern auch um synthetische Funktionen:

- Konzeptualisierung in IT-unterstützt entwickelten und gewarteten Vokabularien bzw. Terminologiedatenbanken, hierarchische Relationen herstellenden Thesauri und Netzwerkstrukturen abbildenden Ontologien schafft Ressourcen für die systematische semantische Analyse bzw. Verarbeitung von Rechtstexten.

- Der Einsatz von Methoden des «Natural Language Processing» zur Aufbereitung großer Korpora von Rechtstexten ermöglicht semantische Annotation zur Unterstützung von Suchalgorithmen und damit neuartige, über die klassische Volltextsuche hinausgehende Suchvorgänge bzw., wenn nicht (nur) mit thematischen Konzepten, sondern mit rechtslogischen Regeln annotiert wird, sogar die Anwendung von «Legal Reasoning»-Systemen.

- Maschinelle Übersetzung von Rechtstexten eröffnet zumindest einen raschen ersten translingualen Zugang zu den Rechtsvorschriften anderer Rechtsordnungen. (Was vor wenigen Jahren noch als eine Quelle nicht enden wollender Heiterkeit erschienen ist, hat sich mittlerweile, wenn gut trainiert, schon zu einem nützlichen Werkzeug entwickelt.)

- Automationsunterstützte sprachliche Transformation bleibt aber nicht auf den translingualen Bereich beschränkt, sie ist auch und umso mehr translektisch einsetzbar: Transformationsysteme zur Reduktion sprachlicher Komplexität können

52 Vgl. z.B. ERICH SCHWEIGHOFER u.a., LOIS: Erfahrungen und Herausforderungen bei der Weiterentwicklung multilingualer Rechtsontologien, in: DERS. u.a. (Hg.), e-Staat und e-Wirtschaft aus rechtlicher Sicht. Tagungsband des 9. Internationalen Rechtsinformatik-Symposions IRIS 2006, Stuttgart 2006, S. 290-295, oder DERS., Juristische Thesauri und Rechtssprache, in: DERS. u.a. (Hg.), Abstraktion und Applikation. Tagungsband des 16. Internationalen RechtsinformatikSymposions IRIS 2013, Wien 2013, S. 389-392. 
die zielgruppenspezifische Rechtsinformation unterstützen, etwa durch reduktionistische Transformation von Rechtstexten in verschiedene Ebenen «Leichter Sprache».

- Schließlich verheißen rechtsinformatische Vorschlags- und Prüfsysteme sogar Assistenz in der Produktion von Rechtstexten: Sie eignen sich beispielsweise dafür, die Einhaltung linguistischer, etwa «klarsprachlicher» Regeln in der Entwurfsarbeit zu unterstützen, aber im Rahmen fortgeschrittener legistischer Arbeitsumgebungen auch für die Erleichterung angewandter Terminologiearbeit.

Der Rechtsinformatik bildet die Rechtssprache somit ein vielfältiges und anspruchsvolles Arbeitsgebiet. Die mit wachsender Rechnerkapazität an Leistungsfähigkeit gewinnenden Methoden des maschinellen Lernens oder der «Künstlichen Intelligenz» erlauben neue Ansätze, die schon heute in Forschungs- und Anwendungsprojekten verfolgt werden.

Das Problem der Verarbeitung von Rechtstexten im europäischen Mehrebenensystem beispielsweise nimmt mit dem Ziel der Unterstützung transjurisdiktionaler und translingualer Recherche nach Rechtsvorschriften durch Einsatz von Methoden des «Natural Language Processing» zur semantischen Anreicherung der in den öffentlichen Rechtsinformationsportalen zugänglichen Ressourcen und zur Erkennung von semantischen Relationen in und zwischen diesen Ressourcen das von der «Connecting Europe Facility» geförderte Projekt «ManyLaws» in den Blick ${ }^{53}$. Aber das ist eine andere Geschichte. Oder sie ist zumindest ein andermal zu erzählen. 